\title{
A GENERALIZATION OF THE THEOREM OF SYLVESTER
}

\author{
FRITZ HERZOG AND L. M. KELLY
}

1. Introduction. The geometric phenomenon which may now properly be referred to as the theorem of Sylvester has received considerable attention during the past decade (see bibliography). This theorem asserts that if a finite number of points in $E_{n}$ (or real projective $n$-space) is such that any line joining a pair of points of the set contains at least three points of the set then the entire set is a subset of a line. For historical remarks and references see [5].

The most recent generalization known to us is that due to Grünbaum [3], in which points in $E_{n}$ are replaced by compact connected sets (continua) in $E_{2}$. More specifically, if $\left\{S_{i}\right\}$ is a finite collection of disjoint plane continua such that any line cutting two distinct sets also cuts some third then the sets are all subsets of the same line.

In this note we weaken the hypotheses and strengthen the conclusion, obtaining a result which may be regarded as, in some sense, the best possible in this direction.

THEOREM. If $\left\{S_{i}\right\}$ is a finite collection of bounded closed disjoint sets in $E_{n}$, at least one of which is infinite, such that any line cutting two distinct sets intersects at least one more, then the sets are all subsets of the same line.

The proof is presented in $\S \S 2$ and 3. The example of a family of three or more parallel lines shows that the assumption of boundedness cannot be dropped. Now consider three disjoint sets $A, B$, and $C$ of the unit interval of the $x$-axis each of which is everywhere dense in the interval. At each point of $A$ erect a unit interval parallel to the $y$-axis and call the union of such segments $A^{*}$. With $B^{*}$ and $C^{*}$ similarly defined, it is clear that a line intersecting any two of the sets must intersect the third, and it follows that the assumption of closure cannot be dropped. Finally, the nine points of the Pappus configuration may be arranged in three sets such that a line cutting any two intersects the third. In particular let $A, B$, and $C$ be three points on one line, $A^{\prime}, B^{\prime}$, and $C^{\prime}$ three points on another, while $A B^{\prime} \cap A^{\prime} B \equiv X, A C^{\prime} \cap A^{\prime} C \equiv Y$, and $B C^{\prime} \cap B^{\prime} C \equiv Z$. The three sets are then $S_{1} \equiv\left\{A, A^{\prime}, Z\right\}, S_{2} \equiv\left\{B, B^{\prime}, Y\right\}, S_{3} \equiv\left\{C, C^{\prime}, X\right\}$. Thus the theorem is not valid without some restriction on the power of the sets.

Received by the editors June 19, 1959. 


\section{The two-dimensional case.}

Definition 2.1. An $E_{n-1}$ in $E_{n}$ which intersects $S$ and is such that at least one of the open half-spaces defined by that $E_{n-1}$ contains at most a finite number of points of $S$ is a pseudo-support $E_{n-1}$ of $S$.

Lemma 2.1. If $\left\{S_{i}\right\}$ is a finite collection of (at least two) disjoint bounded closed sets of the plane with $\cup S_{i} \equiv \Sigma$ infinite, then there exists an index $k, a$ point $P_{k} \in S_{k}^{\prime}$ and a line l through $P_{k}$, which is a pseudosupport line of $\Sigma-S_{k}$ and at least one of whose open half-lines defined by $P_{k}$ is free of points of $\Sigma^{\prime}$.

Proof. There is a support line $\lambda$ of $\Sigma^{\prime}$ which intersects $\Sigma^{\prime}$ in exactly one point, say, $P_{k} \in S_{k}^{\prime}$. This follows from the fact that the convex cover of $\Sigma^{\prime}$ certainly has a support line intersecting the cover in a single point, which point is clearly a point of $\Sigma^{\prime}$. We will suppose $\lambda$ to be horizontal and the open half-plane above $\lambda$ to be free of points of $\Sigma^{\prime}$. If $\lambda$ intersects $\Sigma-S_{k}$ then $\lambda$ is the line $l$ required in the lemma. For, if there were infinitely many points of $\Sigma-S_{k}$ above $\lambda$ then $\Sigma^{\prime}-S_{k}^{\prime}$ would have a point either above $\lambda$ or on $\lambda$, neither of which is possible. If $\lambda$ does not intersect $\Sigma-S_{k}$, then there is a first line $l$ through $P_{k}$, proceeding from $\lambda$ either clockwise or counter-clockwise, which does. This line $l$ then satisfies the requirements of the lemma.

THEOREM 2.1. Let $\left\{S_{i}\right\}$ be a finite collection of disjoint bounded closed subsets of the plane with $\Sigma \equiv \cup S_{i}$ infinite. Then either $\Sigma$ is a subset of a line or there exists a line cutting exactly two of the sets.

Proor. Let $l$ and $P_{k}$ be chosen according to Lemma 2.1. We suppose $l$ to be horizontal, the open half-plane above $l$ to contain at most finitely many points of $\Sigma-S_{k}$, and the open left half-line of $l$ defined by $P_{k}$ to be free of points of $\Sigma^{\prime}$. We consider the following three cases (which are not mutually exclusive).

CASE 1. There exists a sequence of points $\left\{Q_{j}\right\}$ in the half-plane above $l$ with $\lim Q_{j}=P_{k}$. Consider the point $P^{*} \in \Sigma-S_{k}$ on $l$ which is furthest to the right or furthest to the left of $P_{k}$. One of the lines $P^{*} Q_{j}$ meets exactly two of the sets $S_{i}$.

CASE 2. There exists a sequence of points $\left\{Q_{j}\right\}$ in the half-plane below $l$ with $\lim Q_{j}=P_{k}$. (a) If there are points of $\Sigma-S_{k}$ on $l$ to the right of $P_{k}$, let $P^{*}$ be the nearest one of these to $P_{k}$. One of the lines $P^{*} Q_{j}$ meets exactly two of the sets $S_{i}$. (b) If there is no point of $\Sigma-S_{k}$ on $l$ to the right of $P_{k}$, let $P^{*}$ be any point of $\Sigma-S_{k}$ on $l$ to the left of $P_{k}$. Again one of the lines $P^{*} Q_{j}$ meets exactly two of the sets $S_{i}$. 
CASE 3. There exists a sequence of points $\left\{Q_{j}\right\}$ on $l$ with $\lim Q_{j}$ $=P_{k}$; we assume that all $Q_{j}$ are on one side of $P_{k}$. If all of $\Sigma-S_{k}$ lies on $l$, but $S_{k}$ has points not on $l$, then any line through a point of $\Sigma-S_{k}$ and a point of $S_{k}$ not on $l$ will do. We assume that $\Sigma-S_{k}$ has points not on $l$. There is a first line $l^{\prime}$ through $P_{k}$ proceeding counterclockwise from $l$ which has a point in common with $\Sigma-S_{k}$. If $l^{\prime} \cap\left(\Sigma-S_{k}\right)$ has points below $l$, let $P^{*}$ be, among these latter points, the furthest from $P_{k}$ or the nearest to $P_{k}$, according as $\left\{Q_{j}\right\}$ lies to the left or to the right of $P_{k}$, respectively; if $l^{\prime} \cap\left(\Sigma-S_{k}\right)$ has no points below $l$ then let $P^{*}$ be any point of $l^{\prime} \cap\left(\Sigma-S_{k}\right)$. In any one of these cases, one of the lines $P^{*} Q_{j}$ intersects exactly two of the sets $S_{i}$.

\section{The principal theorem.}

Lemma 3.1. Let $\left\{S_{i}\right\}$ be a finite collection of (at least two) disjoint bounded closed subsets of $E_{n}$ with $\Sigma \equiv \cup S_{i}$ infinite and suppose that any $E_{n-1}$ containing points of two different sets contains only a finite number of points of $\Sigma$. Then there exists $a k$ and a pseudo-support $E_{n-1}, \gamma$, of $\Sigma-S_{k}$ with $\gamma \cap S_{k}^{\prime} \neq 0$.

PROOF. We consider a support $E_{n-1}, \alpha$, of $\Sigma^{\prime}$ and suppose that $\alpha$ fails to have the desired property.

CASE 1. $\alpha$ contains points of two distinct sets. We refer to $\alpha$ as horizontal and observe that $\alpha$ contains a point, say $P_{m} \in S_{m}^{\prime}$, as well as a point of a second set $S_{h}, h \neq m$. One of the open half-spaces defined by $\alpha$, say the upper, is free of points of $\Sigma^{\prime}$, but it must contain an infinite number of points of $\Sigma-S_{m}$, otherwise $\alpha$ would have the desired property. Now $\alpha$ contains but a finite number of points of $\Sigma$ and hence there is an $E_{n-2}$, say $\beta$, contained in $\alpha$ which is a support $E_{n-2}$ for $\alpha \cap \Sigma^{\prime}$ in $\alpha$ and which contains exactly one point of $\alpha \cap \Sigma^{\prime}$, say $P_{k} \in S_{k}$. There is a sequence of points $\left\{Q_{j}\right\}$ above $\alpha$ with $\lim Q_{j}$ a point of $\alpha \cap\left(\Sigma-S_{k}\right)^{\prime}$. It is now clear that for some $j$ the $E_{n-1}$ defined by $\beta$ and the point $Q_{j}$ is a pseudo-support $E_{n-1}$ of $\Sigma-S_{k}$ and contains a point $P_{k} \in S_{k}^{\prime}$.

CASE 2. $\alpha$ contains points of but one set $S_{k}$. Again referring to $\alpha$ as horizontal we observe that one of the half-spaces, say the upper, defined by $\alpha$ contains but a finite number of points of $\Sigma-S_{k}$. Consider an $E_{n-2}$, say $\beta$, contained in $\alpha$ with $\beta \cap S_{k}^{\prime} \neq 0$. Now the family of $E_{n-1}$ 's containing $\beta$ has a first element proceeding in either direction from $\alpha$ which contains points of $\Sigma-S_{k}$. Such an element $\gamma$ is a pseudosupport $E_{n-1}$ for $\Sigma-S_{k}$ and intersects $S_{k}^{\prime}$.

Theorem 3.1. Let $\left\{S_{i}\right\}$ be a finite collection of disjoint bounded 
closed subsets of $E_{n}$ with $\Sigma \equiv \cup S_{i}$ infinite. Then either all the sets are subsets of the same line or there exists a line cutting exactly two of the sets.

Proof. We make the inductive assumption that the theorem is true in $E_{n-1}$ and suppose further that $\Sigma$ is not a subset of a line.

If there exists an $E_{n-1}, \alpha$, intersecting two of the sets with $\alpha \cap \Sigma$ infinite, then the sets $\alpha \cap S_{i}$ satisfy the inductive hypothesis and hence there is either a line $l$ in $\alpha$ meeting exactly two of the sets $\alpha \cap S_{i}$ or the sets $\alpha \cap S_{i}$ are all subsets of the same line. In the former case $l$ intersects exactly two of the sets $S_{i}$. In the latter there must be a point of $\Sigma$ not on the line. Such a point and the line define a plane $\pi$. The sets $\pi \cap S_{i}$ satisfy the hypotheses of Theorem 2.1 and hence there is a line cutting exactly two of the sets $\pi \cap S_{i}$ and perforce exactly two of the sets $S_{i}$.

We may now suppose that any $E_{n-1}$ intersecting two of the sets contains a finite number of points of $\Sigma$. Lemma 3.1 assures the existence of $\gamma$, a pseudo-support $E_{n-1}$ for $\Sigma-S_{k}$, intersecting $S_{k}^{\prime}$. One of the open half-spaces defined by $\gamma$ contains at most a finite number of points of $\Sigma-S_{k}$. Call this the upper half-space and consider the case in which there are infinitely many points of $S_{k}$ above $\gamma$. There is then a sequence of points $\left\{Q_{j}\right\}$ above $\gamma$ with $\lim Q_{j}=P_{k} \in S_{k}{ }^{\prime}$. Let $P^{*}$ be a point of $\gamma \cap\left(\Sigma-S_{k}\right)$ furthest from $P_{k}$. If $P^{*} \in S_{m}$ then it is clear that one of the lines $P^{*} Q_{j}$ meets only the sets $S_{k}$ and $S_{m}$.

There remains to consider the case in which there are at most a finite number of points of $\Sigma$ above $\gamma$. In this event, consider the convex cover of $\gamma \cap \Sigma^{\prime}$. This set is, of course, not empty since it contains $P_{k}$. If the only points of $\Sigma$ in this cover are points of $S_{k}$, consider a sequence of points $\left\{Q_{j}\right\}$ below $\gamma$ with $\lim Q_{j}=P_{k}$ together with any point $R \in \gamma \cap\left(\Sigma-S_{k}\right)$. Then one of the lines $R Q_{j}$ will intersect exactly two of the sets.

If the convex cover of $\gamma \cap \Sigma^{\prime}$ contains points of $\Sigma-S_{k}$, let $P_{m}$ be a vertex of this cover, $P_{m} \in S_{m}$, and consider a point $P^{*}$ of the cover, not in the set $S_{m}$, but nearest to $P_{m}$. There is a sequence of points $\left\{R_{j}\right\}$ below $\gamma$ with $\lim R_{j}=P_{m}$. One of the lines $P^{*} R_{j}$ will intersect exactly two of the sets $S_{i}$.

4. The finite case. The example of the Pappus configuration, mentioned in the introduction, shows that it is possible to have three disjoint finite sets such that any line cutting two of the sets cuts the third without the three sets being subsets of the same line. It seems likely to us that, aside from a "few" basic projective configurations, 
Theorem 3.1 can be extended to cover the finite case. However, the best we have been able to do is the following.

THEOREM 4.1. If $\left\{S_{i}\right\}$ is a finite collection of disjoint sets with $\Sigma \equiv U S_{i}$ finite, and any line cutting two of the sets cuts at least two more sets then $\Sigma$ is a subset of a line.

Proof. Let $\Delta(X, Y Z)$ denote the distance from the point $X$ to the line joining the points $Y$ and $Z$. Suppose now that $\Sigma$ is not a subset of any line, and consider the minimum of all positive values of $\Delta(X, Y Z)$ for $X \in \Sigma$ and $Y$ and $Z$ points of two distinct sets $S_{i}$. Let $\Delta(P, Q R)$ be this minimum. Observe that the line $l$, joining $Q$ and $R$ contains points from four different sets, three of which are different from that containing $P$. If $A, B$, and $C$ are three points on $l$ belonging to these three sets then at least two of them, say $A$ and $B$, fall on one of the closed half-lines of $l$ defined by $P^{*}$, the foot of the perpendicular from $P$ to the line $l$. Assuming the order $A B P^{*}$, we see that $0<\Delta(B, A P)$ $<\Delta(P, Q R)$, a contradiction.

Added in proof. In a paper entitled $A$ further generalization of $a$ problem by Sylvester, Riveon Lematematika, 1957, M. Edelstein has extended the result of Grünbaum [3] in a somewhat different direction.

\section{BIBLIOGRAPHY}

1. H. S. M. Coxeter, A problem of collinear points, Amer. Math. Monthly vol. 55 (1948) pp. 26-28.

2. G. A. Dirac, Collinearity properties of sets of points, Quart. J. Math. vol. 2 (1951) pp. 221-227.

3. B. Grünbaum, A generalization of a problem of Sylvester, Riveon Lematematika, 1956.

4. H. Hanani, On the number of lines determined by $n$ points, Technion. Israel Inst. Tech. Sci. Publ. vol. 6 (1954-1955) pp. 58-63.

5. L. M. Kelly and W. O. J. Moser, On the number of ordinary lines determined by $n$ points, Canad. J. Math. vol. 10 (1958) pp. 210-219.

6. Th. Motzkin, The lines and planes connecting the points of a finite set, Trans. Amer. Math. Soc. vol. 70 (1951) pp. 451-464.

Michigan State University 